\title{
DisS IM: A Discourse-Aware Syntactic Text Simplification Framework for English and German
}

\author{
Christina Niklaus $^{13}$, Matthias Cetto ${ }^{1}$, André Freitas ${ }^{2}$, and Siegfried Handschuh ${ }^{13}$ \\ ${ }^{1}$ University of St.Gallen \\ \{christina.niklaus, matthias.cetto, siegfried.handschuh\}@unisg.ch \\ ${ }^{2}$ University of Manchester \\ andre.freitas@manchester.ac.uk \\ ${ }^{3}$ University of Passau \\ \{christina.niklaus, siegfried.handschuh\}@uni-passau.de
}

\begin{abstract}
We introduce DISSIM, a discourse-aware sentence splitting framework for English and German whose goal is to transform syntactically complex sentences into an intermediate representation that presents a simple and more regular structure which is easier to process for downstream semantic applications. For this purpose, we turn input sentences into a twolayered semantic hierarchy in the form of core facts and accompanying contexts, while identifying the rhetorical relations that hold between them. In that way, we preserve the coherence structure of the input and, hence, its interpretability for downstream tasks.
\end{abstract}

\section{Introduction}

We developed a syntactic text simplification (TS) approach that can be used as a preprocessing step to facilitate and improve the performance of a wide range of artificial intelligence (AI) tasks, such as Machine Translation, Information Extraction (IE) or Text Summarization. Since shorter sentences are generally better processed by natural language processing (NLP) systems (Narayan et al., 2017), the goal of our approach is to break down a complex source sentence into a set of minimal propositions, i.e. a sequence of sound, self-contained utterances, with each of them presenting a minimal semantic unit that cannot be further decomposed into meaningful propositions (Bast and Haussmann, 2013).

However, any sound and coherent text is not simply a loose arrangement of self-contained units, but rather a logical structure of utterances that are semantically connected (Siddharthan, 2014). Consequently, when carrying out syntactic simplification operations without considering discourse implications, the rewriting may easily result in a disconnected sequence of simplified sentences that lack important contextual information, making the text harder to interpret. Thus, in order to preserve the coherence structure and, hence, the interpretability of the input, we developed a discourse-aware TS approach based on Rhetorical Structure Theory (RST) (Mann and Thompson, 1988). It establishes a contextual hierarchy between the split components, and identifies and classifies the semantic relationship that holds between them. In that way, a complex source sentence is turned into a so-called discourse tree, consisting of a set of hierarchically ordered and semantically interconnected sentences that present a simplified syntax which is easier to process for downstream semantic applications and may support a faster generalization in machine learning tasks.

\section{System Description}

We present DiSSiM, a discourse-aware sentence splitting approach for English and German that creates a semantic hierarchy of simplified sentences. ${ }^{1}$ It takes a sentence as input and performs a recursive transformation process that is based upon a small set of 35 hand-crafted grammar rules for the English version and 29 rules for the German approach. ${ }^{2}$ These patterns were heuristically determined in a comprehensive linguistic analysis and encode syntactic and lexical features that can be derived from a sentence's parse tree. ${ }^{3}$ Each rule

\footnotetext{
${ }^{1}$ The source code of our framework is available under https://github.com/Lambda-3/ DiscourseSimplification.

${ }^{2}$ For reproducibility purposes, the complete set of transformation patterns is available under https://github. com/Lambda-3/DiscourseSimplification/ tree/master/supplemental_material.

${ }^{3}$ For the English version, we use Stanford's pre-trained lexicalized parser (Socher et al., 2013) to create a sentence's phrasal parse tree. For the German approach, we apply dependency parse structures generated by the spaCy parser (https://spacy.io/).
} 


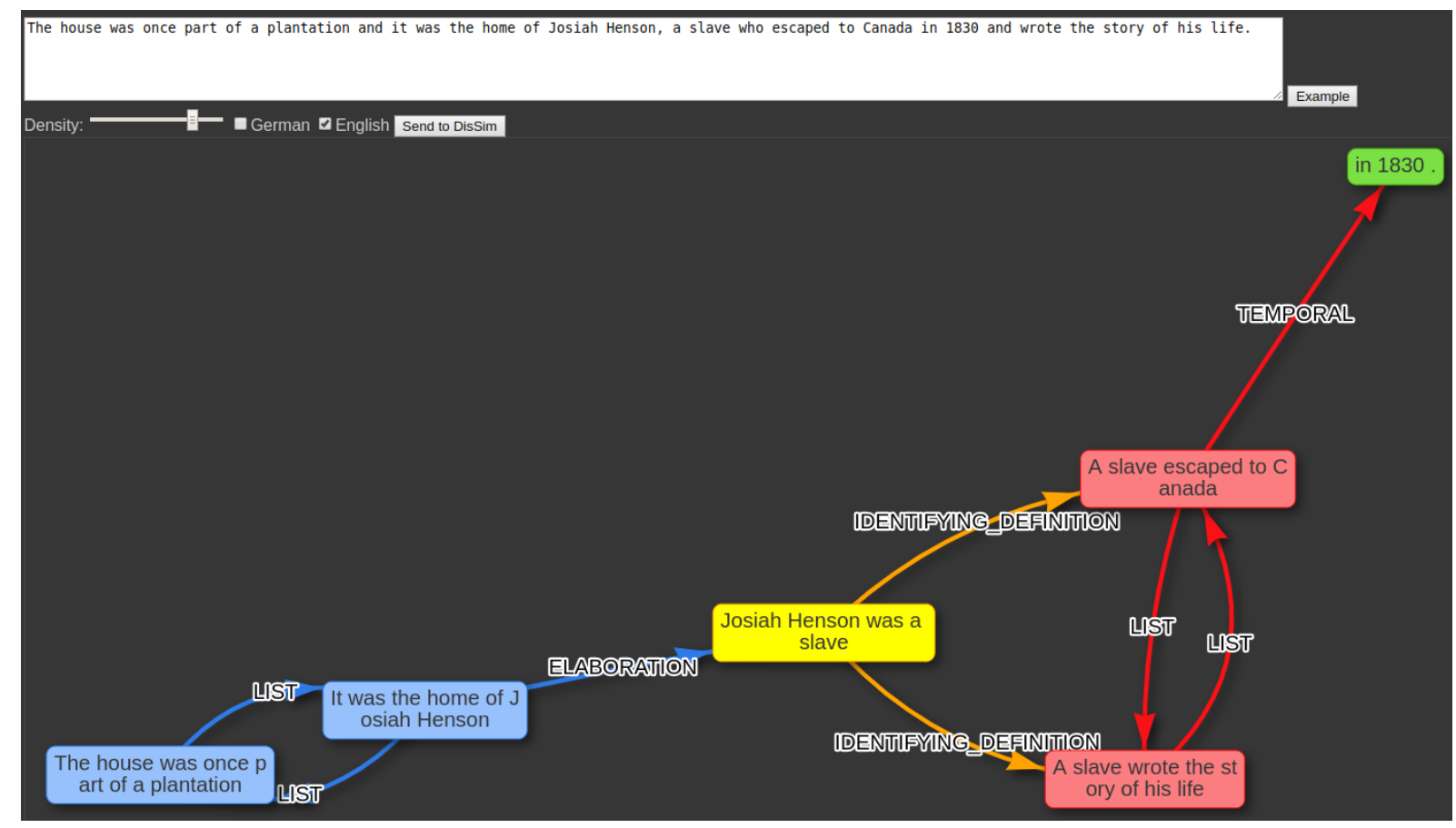

Figure 1: DisSiM's browser-based user interface. The simplified output is displayed in the form of a directed graph where the split sentences are connected by arrows whose labels denote the semantic relationship that holds between a pair of simplified sentences and whose direction indicates their contextual hierarchy. The colors signal different context layers. In that way, a semantic hierarchy of minimal, self-contained propositions is established.

specifies (1) how to split up and rephrase the input into structurally simplified sentences and (2) how to set up a semantic hierarchy between them. They are recursively applied on a given source sentence in a top-down fashion. When no more rule matches, the algorithm stops and returns the generated discourse tree.

\subsection{Split into Minimal Propositions}

In a first step, source sentences that present a complex linguistic form are turned into clean, compact structures by decomposing clausal and phrasal components. For this purpose, the transformation rules encode both the splitting points and rephrasing procedure for reconstructing proper sentences.

\subsection{Establish a Semantic Hierarchy}

Each split will create two or more sentences with a simplified syntax. To establish a semantic hierarchy between them, two subtasks are carried out:

Constituency Type Classification. First, we set up a contextual hierarchy between the split sentences by connecting them with information about their hierarchical level, similar to the concept of nuclearity in RST. For this purpose, we distinguish core sentences (nuclei), which carry the key infor- mation of the input, from accompanying contextual sentences (satellites) that disclose additional information about it. To differentiate between those two types of constituents, the transformation patterns encode a simple syntax-based approach where subordinate clauses/phrases are classified as context sentences, while superordinate as well as coordinate clauses/phrases are labelled as core.

Rhetorical Relation Identification. Second, we aim to restore the semantic relationship between the disembedded components. For this purpose, we identify and classify the rhetorical relations that hold between the simplified sentences, making use of both syntactic features, which are derived from the input's parse tree structure, and lexical features in the form of cue phrases. Following the work of Taboada and Das (2013), they are mapped to a predefined list of rhetorical cue words to infer the type of rhetorical relation.

\section{Usage}

DISSIM can be either used as a Java API, imported as a Maven dependency, or as a service which we provide through a command line interface or a REST-like web service that can be deployed via docker. It takes as input NL text in the form of a 


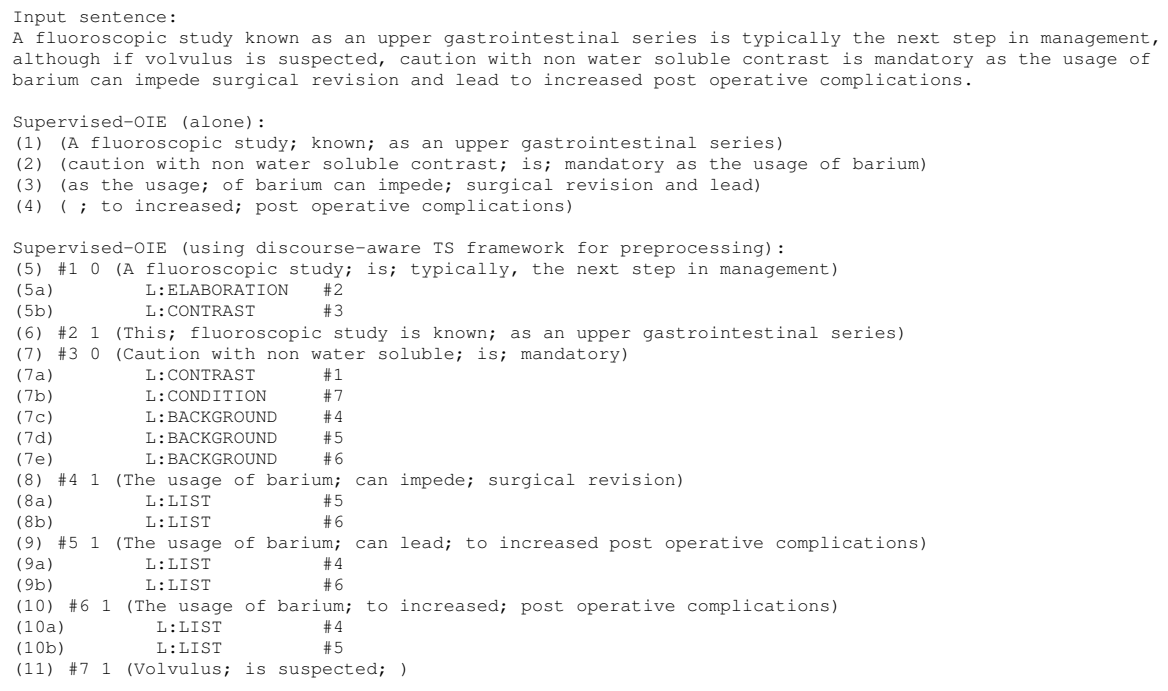

Figure 2: Comparison of the propositions extracted by Supervised-OIE (Stanovsky et al., 2018) with (5-11) and without (1-4) using our discourse-aware TS approach as a preprocessing step.

single sentence. Alternatively, a file containing a sequence of sentences can be loaded. The result of the transformation process is either written to the console or stored in a specified output file in JSON format. We also provide a browser-based user interface, where the user can directly type in sentences to be processed (see Figure 1). ${ }^{4}$

\section{Experiments}

For the English version, we performed both a thorough manual analysis and automatic evaluation across three commonly used TS datasets from two different domains in order to assess the performance of our framework with regard to the sentence splitting subtask. The results show that our proposed sentence splitting approach outperforms the state of the art in structural TS, returning finegrained simplified sentences that achieve a high level of grammaticality and preserve the meaning of the input. The full evaluation methodology and detailed results are reported in Niklaus et al. (2019). In addition, a comparative analysis with the annotations contained in the RST Discourse Treebank (Carlson et al., 2002) demonstrates that we are able to capture the contextual hierarchy between the split sentences with a precision of almost $90 \%$ and reach an average precision of approximately $70 \%$ for the classification of the rhetorical relations that hold between them. The evaluation of the German version is in progress.

\footnotetext{
${ }^{4} \mathrm{~A}$ demonstration video is available online: https : / / streamable.com/08clo.
}

\section{Application in Downstream Tasks}

An extrinsic evaluation was carried out on the task of Open IE (Banko et al., 2007). It revealed that when applying DISSIM as a preprocessing step, the performance of state-of-the-art Open IE systems can be improved by up to $346 \%$ in precision and $52 \%$ in recall, i.e. leading to a lower information loss and a higher accuracy of the extracted relations. For details, the interested reader may refer to Niklaus et al. (2019).

Moreover, most current Open IE approaches output only a loose arrangement of extracted tuples that are hard to interpret as they ignore the context under which a proposition is complete and correct and thus lack the expressiveness needed for a proper interpretation of complex assertions (Niklaus et al., 2018). As illustrated in Figure 2 , with the help of the semantic hierarchy generated by our discourse-aware sentence splitting approach the output of Open IE systems can be easily enriched with contextual information that allows to restore the semantic relationship between a set of propositions and, hence, preserve their interpretability in downstream tasks.

\section{Conclusion}

We developed and implemented a discourse-aware syntactic TS approach that recursively splits and rephrases complex English or German sentences into a semantic hierarchy of simplified sentences. The resulting lightweight semantic representation can be used to facilitate and improve a variety of AI tasks. 


\section{References}

Michele Banko, Michael J. Cafarella, Stephen Soderland, Matt Broadhead, and Oren Etzioni. 2007. Open information extraction from the web. In Proceedings of the 20th International Joint Conference on Artifical Intelligence, pages 2670-2676, San Francisco, CA, USA. Morgan Kaufmann Publishers Inc.

Hannah Bast and Elmar Haussmann. 2013. Open information extraction via contextual sentence decomposition. In 2013 IEEE Seventh International Conference on Semantic Computing, pages 154-159. IEEE.

Lynn Carlson, Mary Ellen Okurowski, and Daniel Marcu. 2002. RST discourse treebank. Linguistic Data Consortium, University of Pennsylvania.

William C Mann and Sandra A Thompson. 1988. Rhetorical structure theory: Toward a functional theory of text organization. Text-Interdisciplinary Journal for the Study of Discourse, 8(3):243-281.

Shashi Narayan, Claire Gardent, Shay B. Cohen, and Anastasia Shimorina. 2017. Split and rephrase. In Proceedings of the 2017 Conference on EMNLP, pages 606-616. ACL.

Christina Niklaus, Matthias Cetto, André Freitas, and Siegfried Handschuh. 2018. A survey on open information extraction. In Proceedings of the 27th International Conference on Computational Linguistics, pages 3866-3878, Santa Fe, New Mexico, USA. Association for Computational Linguistics.

Christina Niklaus, Matthias Cetto, André Freitas, and Siegfried Handschuh. 2019. Transforming complex sentences into a semantic hierarchy. In Proceedings of the 57th Annual Meeting of the Association for Computational Linguistics, pages 3415-3427, Florence, Italy. Association for Computational Linguistics.

Advaith Siddharthan. 2014. A survey of research on text simplification. ITL-International Journal of Applied Linguistics, 165(2):259-298.

Richard Socher, John Bauer, Christopher D. Manning, and Andrew Y. Ng. 2013. Parsing With Compositional Vector Grammars. In $A C L$.

Gabriel Stanovsky, Julian Michael, Luke Zettlemoyer, and Ido Dagan. 2018. Supervised open information extraction. In Proceedings of the 2018 Conference of the North American Chapter of the Association for Computational Linguistics: Human Language Technologies, Volume 1 (Long Papers), volume 1, pages 885-895.

Maite Taboada and Debopam Das. 2013. Annotation upon annotation: Adding signalling information to a corpus of discourse relations. $D \& D, 4(2): 249-281$. 